\title{
Cultura tributaria y evasión de impuestos de las Pymes en la ciudad de Moyobamba - San Martin
}

\author{
Tax culture and tax evasion of SMEs in the city of Moyobamba - San Martin
}

\begin{tabular}{rrr} 
Edwin Mori & Vidalina Tineo & Elisa Tocto Herrera \\
\hline edwinmori@upeu.edu.pe & vidalinatineo@upeu.edu.pe & viluzmar_vida18@hotmail.com \\
ORCID: 0000-0001-6740-5473 & ORCID: 0000-0001-5063-6929 & ORCID: 0000-0003-3511-7586 \\
Universidad Peruana Unión & Universidad Peruana Unión & Universidad Peruana Unión
\end{tabular}

Artículo recibido en abril 2019 / Arbitrado en mayo 2019 / Publicado en julio 2019

RESUMEN | El presente trabajo de investigación es de suma importancia; ya que pretende conocer sobre la evasión de impuestos de las Pymes en la ciudad de Moyobamba - San Martín 2020, viendo de qué manera se relaciona con la cultura tributaria. Tuvo como objetivo determina la relación entre la cultura tributaria y evasión de impuestos, de las Pymes en la ciudad de Moyobamba- San Martin 2020. El diseño asumido para la presente investigación es de no experimental, y el tipo de investigación está catalogada como descriptiva. Según los resultados los contribuyentes no califican como bueno la información brindada por la SUNAT para prevenir las infracciones y sanciones de los contribuyentes en la ciudad de Moyobamba, es decir un el 9.1\% lo califica como malo, podemos observar aquí que los contribuyentes no ven como medio de apoyo a la SUNAT que les pueda brindar una información según sus necesidades o no está llegando a comunicar bien.

Palabras clave SUNAT, impuestos, evasión, tributario, Pymes

\footnotetext{
ABSTRACT | This research work is of utmost importance; since it intends to know about the tax evasion of SMEs in the city of Moyobamba - San Martín 2020, seeing how it is related to the tax culture. Its objective was to determine the relationship between the tax culture and tax evasion of SMEs in the city of Moyobamba- San Martin 2020. The design assumed for this research is non-experimental, and the type of research is classified as descriptive. According to the results, the taxpayers do not qualify as good the information provided by SUNAT to prevent the infractions and sanctions of the taxpayers in the city of Moyobamba, that is, $9.1 \%$ qualify it as bad, we can observe here that the taxpayers do not see how means of support to SUNAT that can provide them with information according to their needs or is not communicating well.

Keywords

SUNAT, taxes, evasion, tributary, SMEs
} 


\section{INTRODUCCIÓN}

La alta evasión tributaria es uno de los rasgos más característicos de la sociedad peruana, desconocen a cabalidad sus obligaciones tributarias con el Estado peruano, en consecuencia, que no pagan los impuestos. Las razones que carecen los conocimientos tributarios que las empresas no cuenta con el conocimiento tributario es por eso que sirven de argumento para el impago son diversas como: no tiene conocimiento tributario, carecen de tiempo y se olvidan de pagar.

Esta es la razón por la que esta investigación realiza este trabajo que resulta ser muy importante en donde se busca desarrollar una mayor cultura tributaria, haciendo de las Pymes en la ciudad de Moyobamba- San Martin, se comprometan e implanten la conciencia tributaria; así como el cumplimiento eficiente de sus funciones de SUNAT, para que haga mejor esta labor

Existe gran evasión tributaria en la ciudad de Moyobamba por parte de las Pymes, ya sea en forma voluntaria o involuntaria. La cultura tributaria de acuerdo al nivel de conciencia referente a los deberes $y$ derechos origina para los individuos activos y pasivos del vínculo tributario. La cultura tributaria y su influencia en la evasión de impuestos, se puede definir que hay una cultura escaza, y esto se refleja en el incumplimiento de las normas tributarias; a causa de que no poseen los conocimientos básicos sobre las obligaciones tributarias.

Por tal razón, la investigación tuvo como objetivo determinar la relación entre de la cultura tributaria y evasión de impuestos en las Pymes de la ciudad de Moyobamba del departamento de San Martin durante el año
2020. Del mismo modo se busca determinar la relación entre la conciencia tributaria en la evasión de impuestos en las Pymes de la ciudad de Moyobamba -San Martin 2020.

Para realizar la investigación, se ha recurrido a otros trabajos similares con temas que tienen relación, por consiguiente, se presentan las siguientes investigaciones:

Bedoya Ramos y Andrea Elizabeth Ecuador, Quito (2012) en su tesis "Determinantes de la evasión tributaria. Una aproximación a través de la economía experimental" realizada y presentada en la Facultad Latinoamericana de Ciencias Sociales para obtener el título de Maestría en Ciencias Sociales con Mención al Desarrollo con el objetivo general de determinar los factores de la evasión tributaria que sucede a través de la economía experimental para los bienes públicos y su metodología de la investigación de los experimentos económicos de laboratorio para demostrar las correlaciones existentes entre la distribución de la renta, la probabilidad de auditoria, la provisión de bienes públicos, los mensajes de cumplimiento y la evasión tributaria.

Por tu parte, Gómez Sebaini y Moran, (2016) dice que la evasión tributaria o evasión de impuestos, es el cumplimiento en la aplicación de una norma tributaria, ya que genera una actividad ilícita, por lo tanto, es un delito y un nivel de informalidad que consiste en ocultar bienes o ingresos es decir pagar menos impuestos. (p.4)

Son hechos de incumplimiento que toda ocasión deshonra las leyes tributarias que produce la eliminación de la carga tributaria. La evasión se expresa como un delito en nuestro 
ordenamiento legal, es así que está ocasionando menor ingreso fiscal. (Villegas, 2017, p.255)

\section{MATERIALES Y MÉTODO}

El diseño asumido para la presente investigación es de no experimental debido a que no se va a manipula las variables. $Y$ desde el punto de vista de diseño es de corte: transversal ya que solo se recogerán los datos en solo momento.

(Hernández, 2014) Define a la investigación no - experimental como "La investigación que se realiza sin manipular deliberadamente variables. Es decir, se trata de estudios donde no hacemos variar en forma intencional las variables independientes para ver su efecto sobre otras variables."

El tipo de investigación es descriptiva, debido a que se enfocara a conocer las variables según el comportamiento que las mismas presenten en el transcurso de la presente investigación.

En este caso la población está conformada la ciudad de Moyobamba, pues los microempresarios de pequeñas y medianas empresas nos brindaran la información necesaria para la investigación. En cuanto a la muestra, Quezada, L. (2012) sostiene que representa una distinción sin ninguna particularidad al elegir de una parte de la población, de todo esto se estudian características con una gran particularidad, por lo general poseen toda una serie de cualidades en común.

Es por ello, que mediante una solicitud ingresada a la SUNAT; Atención a solicitud de transparencia y acceso a la información pública Formulario 5030 N. ${ }^{\circ}$ 88025156, entregó la información mediante correo electrónico sobre la lista de contribuyentes. Finalmente, la SUNAT informó que, a través de la página web de la Sunat, cuya dirección es http://www.sunat.gob.pe 0 ingresando directamente a través del enlace http://www.sunat.gob.pe/descargaPRR/mrc1 37_padron_reducido.html, puede obtener información sobre la relación de contribuyentes inscritos en el RUC.

El total de contribuyentes a nivel de la ciudad de Moyobamba según Sunat en su lista de contribuyentes son un total de 2,509 contribuyentes en el Mype tributario, así mismo para la toma de muestra se está tomando una calculadora de muestra en https://www.corporacionaem.com/tools/calc_ muestras.php.

Se está calculando con un margen del $10 \%$ y un nivel de confianza de $90 \%$ de dicha población y teniendo como resultado una muestra de 66 contribuyentes para encuestar.

Las técnicas de recolección de datos que se aplicaron en la investigación son:

- Encuestas: Se aplicaron como técnica de investigación, puesto que esta técnica hizo posible contactar con los microempresarios de las pequeñas y medianas empresas el instrumento que se aplicó fue el cuestionario.

- Cuestionarios: Se aplicó con la finalidad de medir el nivel de cultura tributaria y el nivel de evasión de impuesto. Estos cuestionarios incluyeron ítems que permite obtener información cualitativa con el nivel de cultura y evasión de impuesto de los encuestados 


\section{RESULTADOS Y DISCUSIONES}

La encuesta fue hecha en el formulario google drive, generando un link corto para que puedan acceder a responder la encuesta mediante su computadora Tablet o celular personal, mediante el link: https://forms.gle/NxKcCsNADkz3mRHJ9.
Dichas encuestas recibidas en el google Drive fueron reportadas por el excel de google forms para poder trabajar las estadísticas y realizar las interpretaciones correspondientes a cada pregunta formulada según la su variable, obteniendo los siguientes resultados.

Grafico 1. Como considera usted la importancia que tiene las Pymes en la economía peruana:

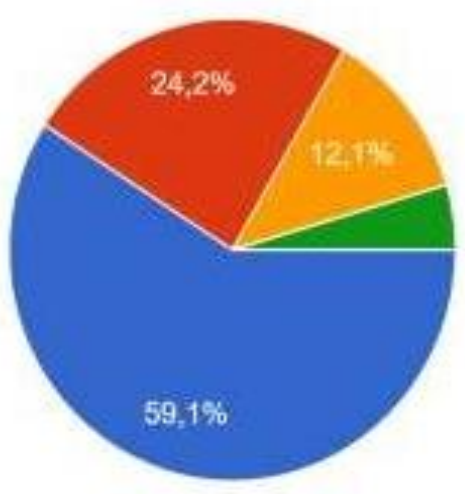

Muy bueno

Bueno

Regular

Malo

Muy malo

Fuente: Mori, Tineo, Tocto (2018)

Respuesta: Se puede observar que el $59.1 \%$ de los encuestados en la provincia de Moyobamba considera muy bueno que las Pymes tienen importancia en la economía peruana y el $24.2 \%$ considera bueno y el $12.1 \%$ considera regular.

Grafico 2. ¿Cómo califica su conocimiento sobre la importancia de la tributación?

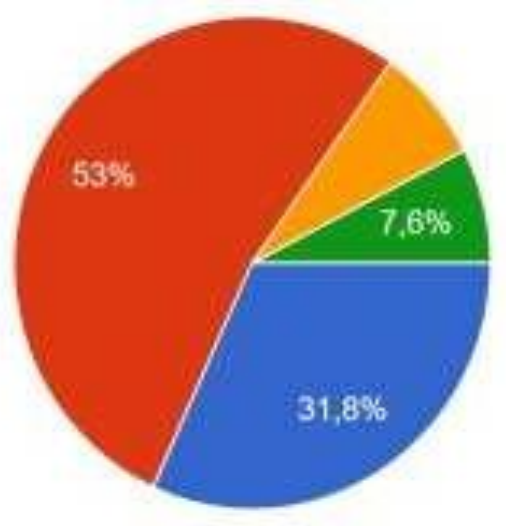

Muy bueno

Bueno

Regular

Malo

Muy malo

Fuente: Mori, Tineo, Tocto (2018)

Respuesta: Se puede observar que el $53 \%$ califica bueno su conocimiento sobre la tributación y el $31.8 \%$ lo considera muy Bueno y el $7.6 \%$ lo considera malo. 
Grafico 3. ¿Cómo considera usted el ciclo de vida de una Pyme en el Perú?

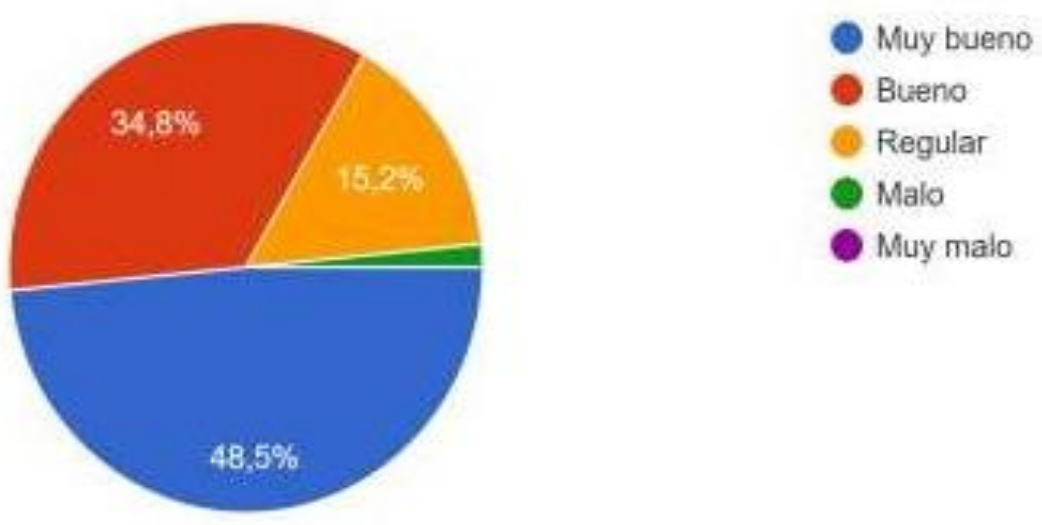

Fuente: Mori, Tineo, Tocto (2018)

Respuesta: Se puede observar que el $48.5 \%$ califica muy bueno su ciclo de vida las Pymes en el Perú, el $34.8 \%$ lo considera bueno y el $15.2 \%$ lo considera regular.

Grafico 4. ¿Qué opina usted sobre contratar familiares y amigos en su negocio?

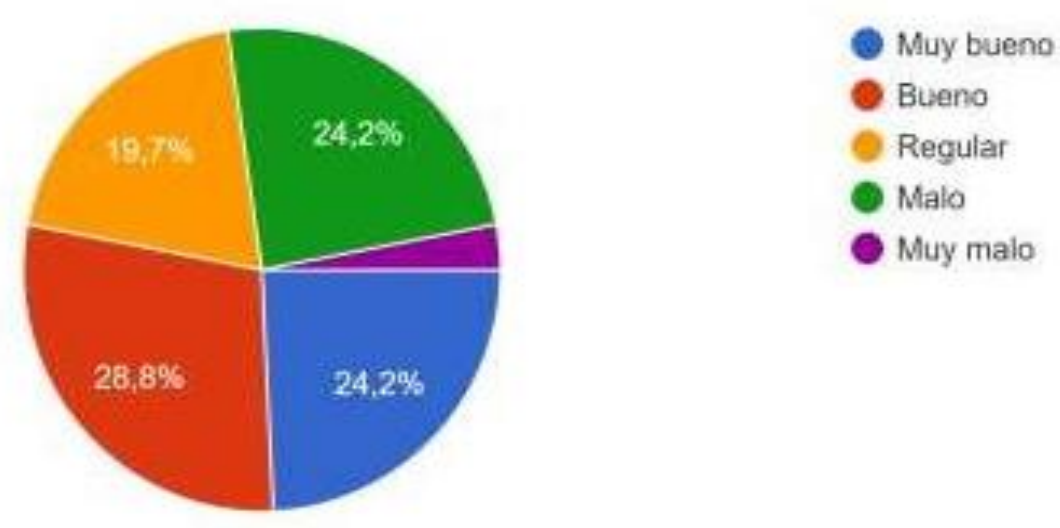

Fuente: Mori, Tineo, Tocto (2018)

Respuesta: Se puede observar que el $28.8 \%$ opina que es bueno contratar a familiares y amigos en su negocio, el $24.2 \%$ lo considera muy bueno y malo. 
Grafico 5. ¿Cómo calificaría la informalidad en la sociedad peruana?

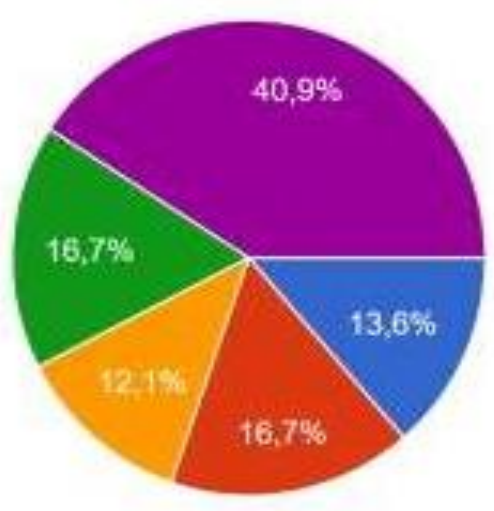

Muy bueno

Bueno

Regular

Malo

Muy malo

Fuente: Mori, Tineo, Tocto (2018)

Respuesta: Se puede observar que el $49.9 \%$ califica como muy malo la informalidad en la sociedad peruana, el $16.7 \%$ está entre malo y bueno y el $13.6 \%$ lo considera muy bueno.

Grafico 6. ¿Cuál es tu conocimiento sobre los alcances de la tributación para el desarrollo del país?

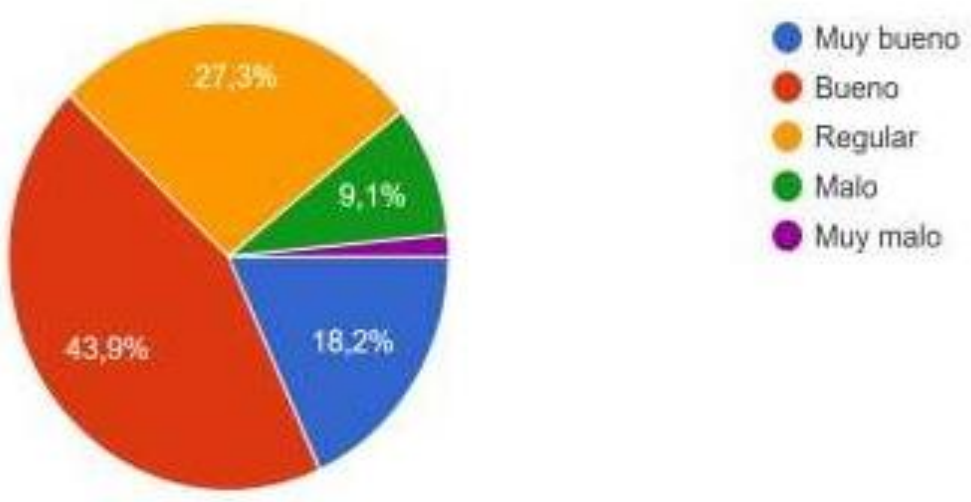

Fuente: Mori, Tineo, Tocto (2018)

Respuesta: Se puede observar que el $43.9 \%$ califica como bueno sus conocimientos sobre los alcances de la tributación para el desarrollo del país, el $27.3 \%$ califica regular sobre sus conocimientos, el $18.2 \%$ entre muy bueno y el $9.1 \%$ lo califica como malo. 
Grafico 7. ¿Cómo califica usted la educación tributaria de los contribuyentes en la ciudad de Moyobamba?

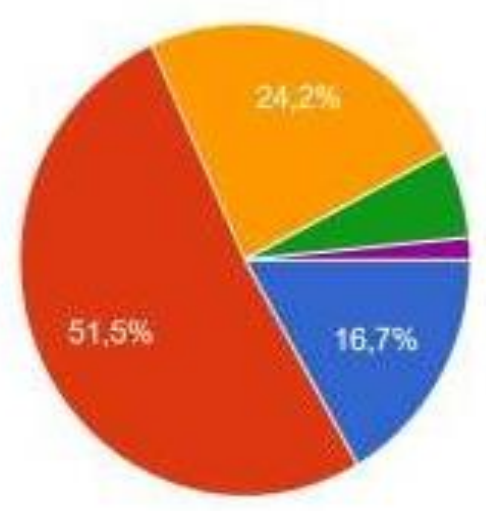

Muy bueno

Bueno

Regular

Malo

Muy malo

Fuente: Mori, Tineo, Tocto (2018)

Respuesta: Se puede observar que el $51.5 \%$ califica como bueno su educación tributaria sobre los contribuyentes en la ciudad de Moyobamba, el $24.2 \%$ califica como regular y el $16.7 \%$ entre muy bueno y el $9.1 \%$ lo califica como malo.

Grafico 8. 10. ¿Cómo califica usted al conocimiento de normas y leyes tributarias de parte en las empresas de la ciudad de Moyobamba?

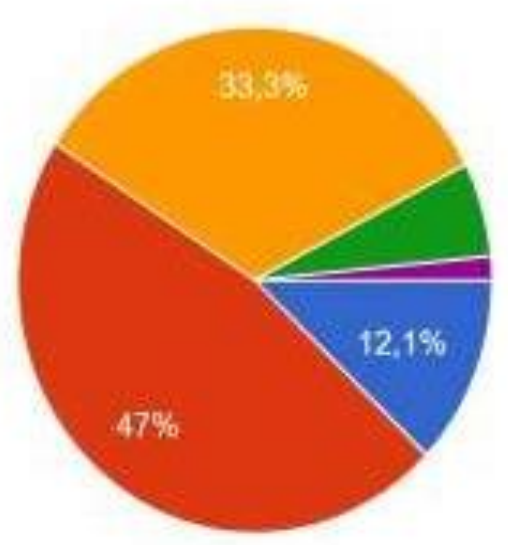

Muy bueno

Bueno

Regular

Malo

Muy maio

Fuente: Mori, Tineo, Tocto (2018)

Respuesta: Se puede observar que el $47 \%$ califica como bueno su conocimiento de normas y leyes tributarias de parte de las empresas de la ciudad de Moyobamba, el 33.3\% califica como regular y el $12.1 \%$ entre muy bueno. 
Grafico 9. ¿Cómo califica usted a SUNAT para contribuir con la formalización en las empresas de la ciudad de Moyobamba?

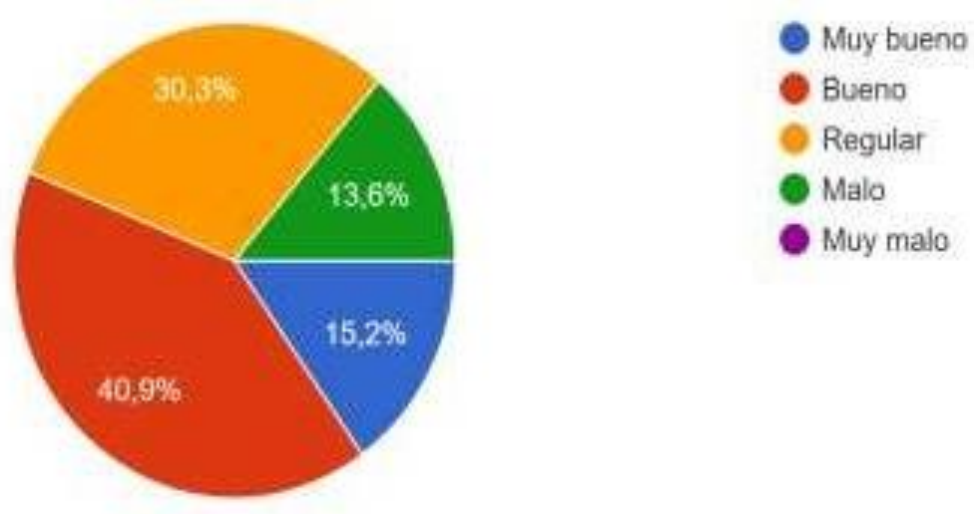

Fuente: Mori, Tineo, Tocto (2018)

Respuesta: Se puede observar que el $40.9 \%$ califica como bueno a la SUNAT para contribuir con la formalización en las empresas de la ciudad de Moyobamba, el 30.3\% califica como regular, el $15.2 \%$ lo califica muy bueno y el $13.6 \%$ lo califica como malo.

Grafico 10. ¿Cómo califica la información tributaria brindada por la SUNAT para evitar y/o prevenir las infracciones y sanciones de los contribuyentes en la ciudad de Moyobamba?

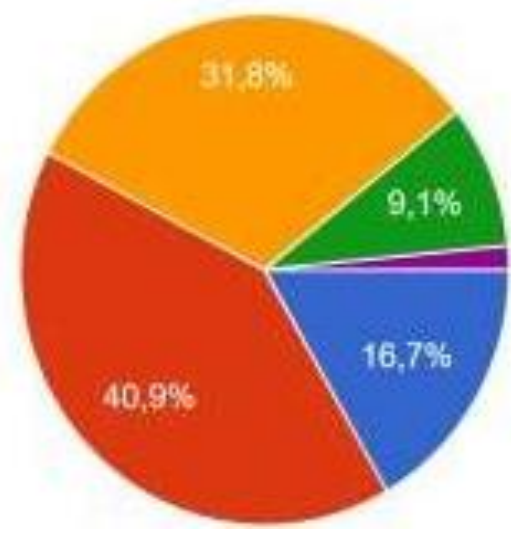

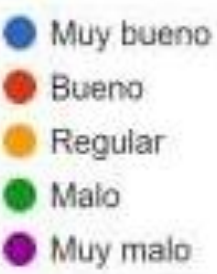

Muy malo

Fuente: Mori, Tineo, Tocto (2018)

Respuesta: Se puede observar que el $40.9 \%$ califica como bueno la información brindada por la SUNAT para prevenir las infracciones y sanciones de los contribuyentes en la ciudad de Moyobamba, el $31.8 \%$ califica como regular, el $16.7 \%$ lo califica muy bueno y el $9.1 \%$ lo califica como malo. 
Grafico 11. ¿Cómo califica usted al cierre de locales por no entregar comprobantes de pago en los contribuyentes de la ciudad de Moyobamba?

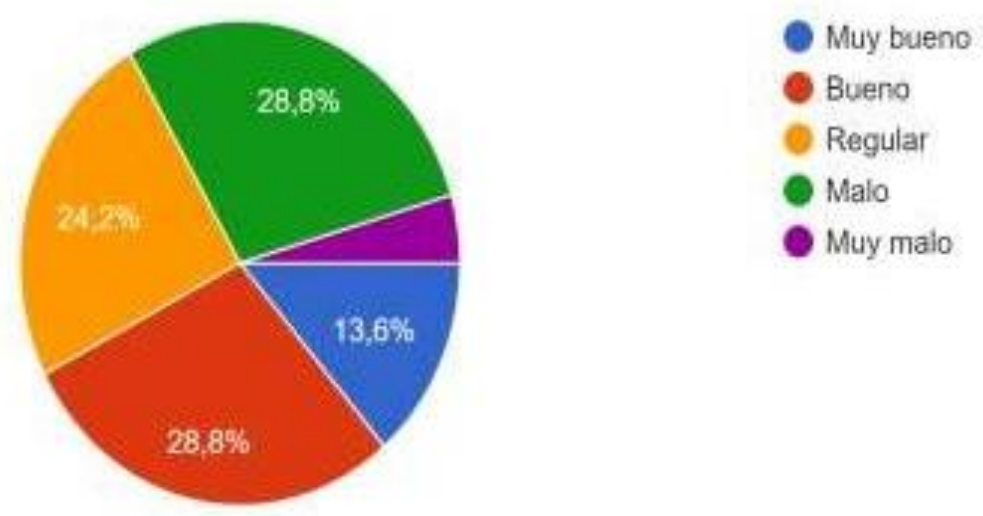

Fuente: Mori, Tineo, Tocto (2018)

Respuesta: Se puede observar que el $28.8 \%$ califica como bueno y malo el cierre de los locales por no entregar comprobantes de pago en los contribuyentes en la ciudad de Moyobamba, el $24.2 \%$ califica como regular, y el $13.6 \%$ lo califica como muy bueno.

Grafico 12. ¿Cómo califica usted las declaraciones de ingresos y gastos de los contribuyentes en la ciudad de Moyobamba?

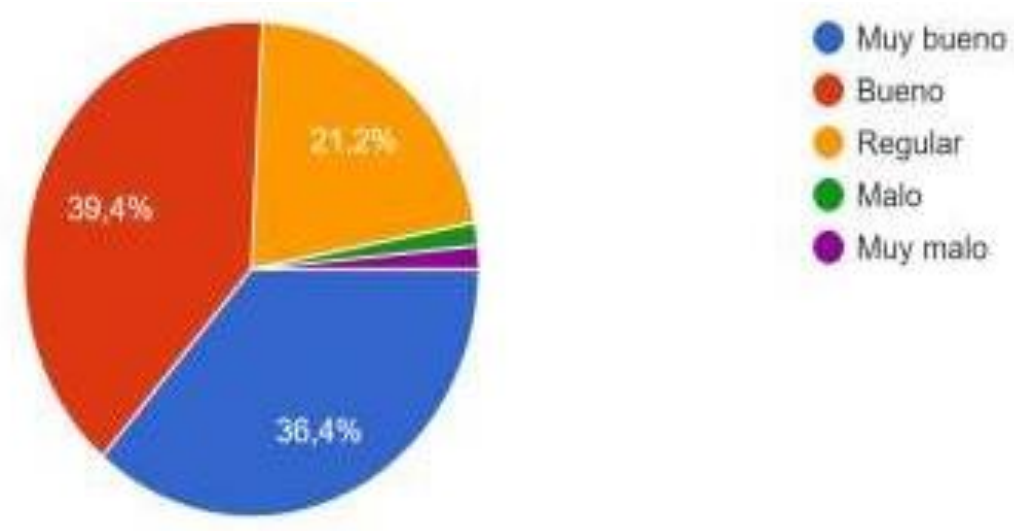

Fuente: Mori, Tineo, Tocto (2018)

Respuesta: Se puede observar que el 39.4\% califica como bueno las declaraciones de ingresos y gastos de los contribuyentes en la ciudad de Moyobamba, el $36.4 \%$ califica como muy bueno y el $21.2 \%$ lo califica como regular. 
Grafico 13. ¿Cómo considera usted la evasión que realizan los contribuyentes de las Pymes y su incidencia en la recaudación tributaria?



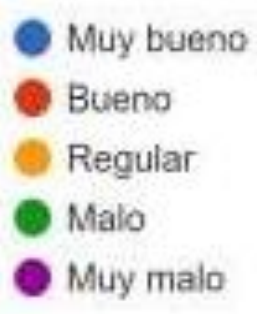

Muy malo

Fuente: Mori, Tineo, Tocto (2018)

Respuesta: Se puede observar que el 57.6\% considera como muy malo la evasión que realizan los contribuyentes de las PYMES y su incidencia en la recaudación tributaria y el $25.8 \%$ lo califica como malo.

Grafico 14. ¿Cómo considera Ud. ¿La declaración y pago de sus impuestos en su negocio?
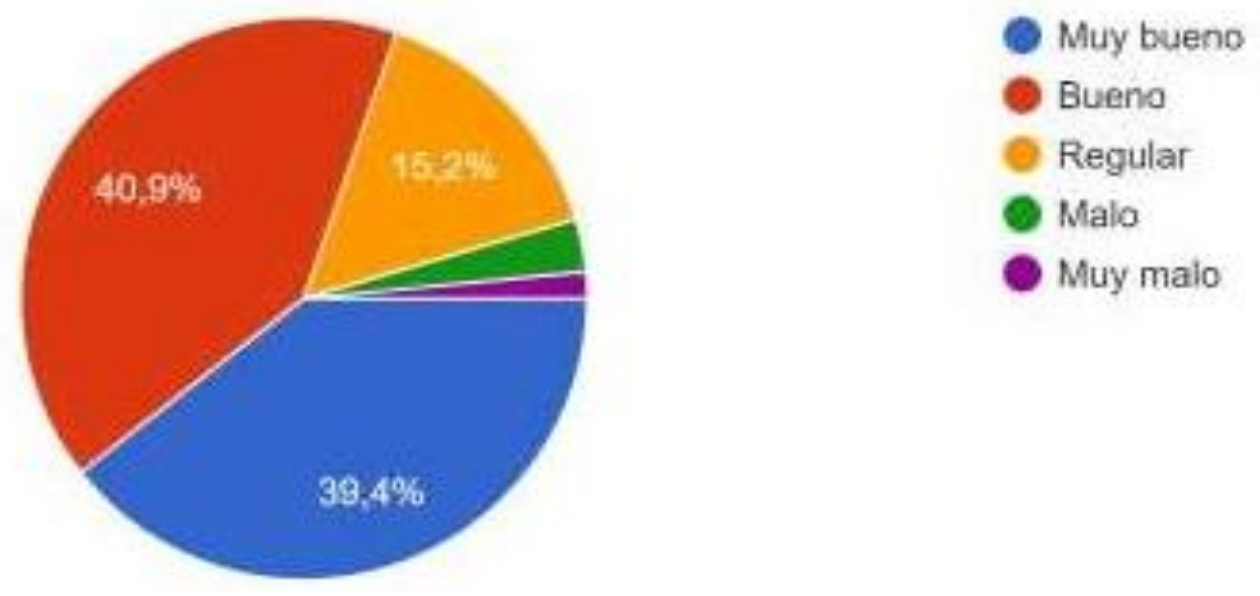

Fuente: Mori, Tineo, Tocto (2018)

Respuesta: Se puede observar que el $40.9 \%$ considera bueno que la declaración y pago de sus impuestos en su negocio, el $39.4 \%$ considera muy bueno y el $15.2 \%$ lo considera como regular. 
Grafico 15. ¿Cómo cree usted que la SUNAT al realizar alguna intervención y operativo a los negocios con frecuencia es?

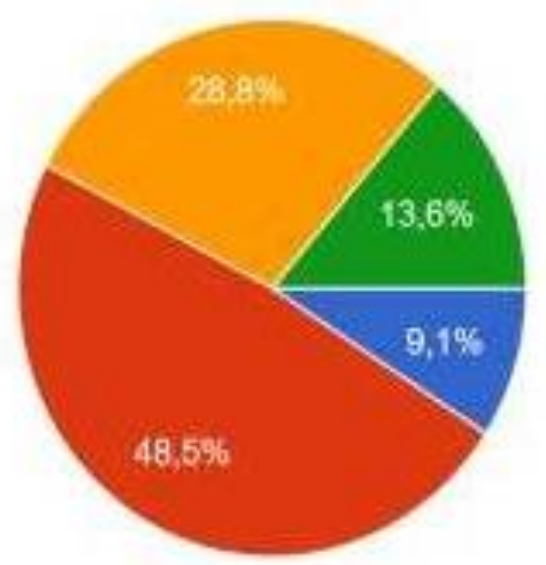

Muy bueno

Bueno

Regular

Malo

Muy majo

Fuente: Mori, Tineo, Tocto (2018)

Respuesta: Se puede observar que el $48.5 \%$ cree que es bueno que la SUNAT realice alguna intervención y operativo a los negocios con frecuencia, el $28.8 \%$ considera regular, el $13.6 \%$ cree que es malo y el $9.1 \%$ lo considera como muy bueno.

Grafico 16. ¿Su negocio ha sido fiscalizado y/o inspeccionado alguna vez por la SUNAT? ¿Cómo lo percibió?

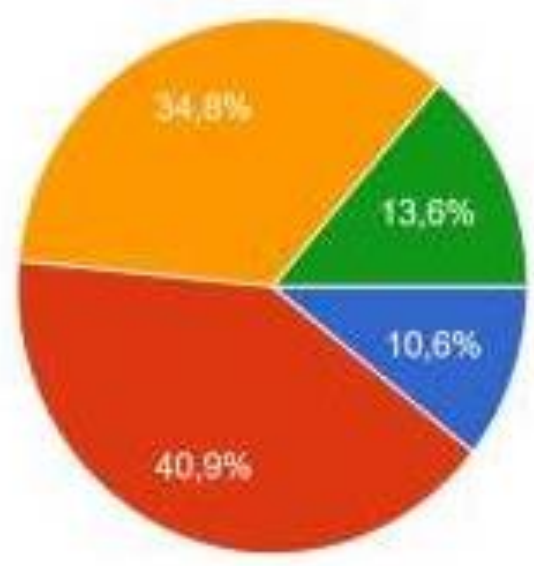

$$
\begin{aligned}
& \text { Muy bueno } \\
& \text { Bueno } \\
& \text { Regular } \\
& \text { Malo } \\
& \text { Muy malo }
\end{aligned}
$$

Fuente: Mori, Tineo, Tocto (2018)

Respuesta: Se puede observar que el $40.9 \%$ ha percibido como bueno cuando su negocio ha sido fiscalizado o intervenido alguna vez por la SUNAT, el 34.8\% lo percibió regular, el 13.6\% lo percibió como malo y el $10.6 \%$ lo percibió como muy bueno. 
Grafico 17. ¿Cómo califica usted la emisión de comprobantes de pago en su negocio?

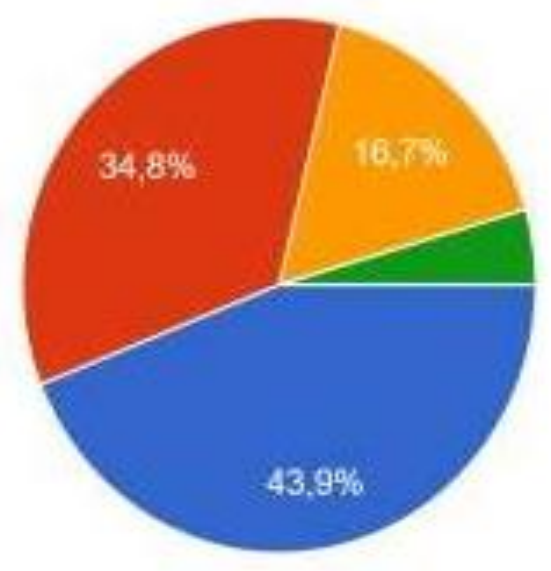

- Muy bueno

- Bueno

(1) Regular

- Malo

- Muy malo

Fuente: Mori, Tineo, Tocto (2018)

Respuesta: Se puede observar que el $43.9 \%$ califica como muy bueno la emisión de comprobantes de pago en su negocio, el $34.8 \%$ lo califica como bueno, el $16.7 \%$ lo califica como regular.

Grafico 18. ¿Cómo considera usted la orientación tributaria por parte de la SUNAT que ha recibido usted?



Muy bueno

Bueno

Regular

Malo

Muy malo

Fuente: Mori, Tineo, Tocto (2018)

Respuesta: Se puede observar que el $40.9 \%$ considera que la orientación tributaria por parte de la SUNAT es buena, el $27.3 \%$ lo percibe como regular, el $24.2 \%$ lo califica como muy bueno y el $7.6 \%$ lo percibe como malo. 
Los resultados nos muestran que el 53\% califica bueno su conocimiento sobre la tributación y el 31.8\% lo considera muy Bueno y el $7.6 \%$ lo considera malo, así mismo el $49.9 \%$ califica como muy malo la informalidad en la sociedad peruana, el $16.7 \%$ está entre malo y bueno y el $13.6 \%$ lo considera muy bueno, es decir los contribuyentes no están de acuerdo con la informalidad ya que muchos de ellos si tienen un buen o regular conocimiento sobre tributación.

En los resultados, también se puede observar que el $51.5 \%$ califica como bueno su educación tributaria sobre los contribuyentes en la ciudad de Moyobamba, el 24.2\% califica como regular y el $16.7 \%$ entre muy bueno y el $9.1 \%$ lo califica como malo, ya que consideran según la encuesta realizada un $43.9 \%$ califica como muy bueno la emisión de comprobantes de pago en su negocio, el $34.8 \%$ lo califica como bueno, el $16.7 \%$ lo califica como regular.

Además, según los resultados los contribuyentes no califican como bueno la información brindada por la SUNAT para prevenir las infracciones y sanciones de los contribuyentes en la ciudad de Moyobamba, es decir un el $9.1 \%$ lo califica como malo, podemos observar aquí que los contribuyentes no ven como medio de apoyo a la SUNAT que les pueda brindar una información según sus necesidades o no está llegando a comunicar bien. Según la investigación en cuanto a la orientación Tributaria brindada por la SUNAT solo un $40.9 \%$ de la población lo considera buena, existiendo un $7.6 \%$ que no lo considera bien sino como mala.

\section{CONCLUSIONES}

La SUNAT tiene un papel muy importante en cuanto a información y fiscalización de los contribuyentes para poder formalizar y tener bien informados a los contribuyentes especialmente a las Pymes, ya que muchas veces por falta de información o desconocimiento no llevan muy bien su contabilidad.

Los contribuyentes de la Mype tributario en la ciudad de Moyobamba deben de mejorar su nivel de cultura tributaria considerando que la informalidad y otras formas de eludir sus responsabilidades tributarias no son normales, saber que lo recaudado tiene un destino adecuado y darle la debida importancia a la difusión sobre tributos y normas que les permita tener conocimiento sobre cultura, conciencia y educación tributaria, informalidad, formalización, deberes y derechos del contribuyente.

Los contribuyentes de la Mype tributario en la ciudad de Moyobamba deben educarse más sobre aspectos tributarios, hecho que les permitirá tomar conocimiento desde la etapa inicial de su formación educativa, sujeta en forma permanente a los valores personales, la tributación, la recaudación y el destino de los fondos recaudados por el Estado. Todo este proceso no sólo produciría educación tributaria sino también conciencia para aceptar las normas tributarias, consecuentemente llegar a contribuir con sus obligaciones tributarias en forma normal. 


\section{REFERENCIAS}

Andra Bedoya. (2011). determinantes de la evasión tributaria. una aproximación a través de la economía experimental. 16(22), 1-97

https://repositorio.flacsoandes.edu.ec/bitstrea m/10469/3880/1/TFLACSO-2011AEBR.pdf Francia y Cristina. (2017). La evasión tributaria y su efecto en la rentabilidad de los restaurantes del distrito de Zalaverry, Trujillo año 2017. Normas Tributarias, 84

Hernandes. (2014). MOTOLOGÍA DE LA INVESTIGACIÓN

http://repositorio.unap.edu.pe/bitstream/handl e/UNAP/1891/Casas_Ochochoque_Joel_Ra inier. . pdf? sequence $=1$ \&isAllowed $=y$

Huatay, L., \& Sánchez, A. (2018). "Influencia de la Cultura Tributaraia en el Cumplimiendo de las obligaciones Tributarias en los
Comerciantes del Mercado San Antonio Cajamarca, Julio 2018". (Tesis de pregrado). Universidad Privada del Norte, Cajamarca, Perú

Amasifuen, R. (2015). Importancia de la cultura tributaria en el Perú. Revista Accounting power for business, 1(1), 73-90

Burga, M. (2015). Cultura tributaria y obligaciones tributarias en las empresas comerciales del Emporio Gamarra de la ciudad de Lima

Uaman, C. y. (2016). Cultura Tributaria y sus obligaciones tributarias en los microempresarios del Huequito $\mathrm{N}^{\circ} 1$ http://observatorio.epacartagena.gov.co/wp -content/uploads/2017/08/metodologiade-la investigacion-sextaedicion.compressed.pdf 